\title{
Evidence for a Potential "Knee-Eye-Brain Axis" Involved in Mobility and Navigation Control: Knee Injury and Obesity May Disrupt Axis Integrity
}

\author{
David A. Hart ${ }^{1,2,3,4}$
}

${ }^{1}$ Faculty of Kinesiology, University of Calgary, Calgary, Alberta, Canada; ${ }^{2}$ Department of Surgery, University of Calgary, Calgary, Alberta, Canada; ${ }^{3}$ McCaig Institute for Bone \& Joint Health, University of Calgary, Calgary, Alberta, Canada; ${ }^{4}$ Bone \& Joint Health Strategic Clinical Network, Alberta Health Services, Alberta, Canada

Correspondence to: David A. Hart, hartd@ucalgary.ca

Keywords: Knee Injury, Eye, Cornea, Vitreous Humor, Vitreous Humor Cells, Obesity

Received: February 12, 2018 Accepted: March 19, $2018 \quad$ Published: March 22, 2018

Copyright $\odot 2018$ by authors and Scientific Research Publishing Inc.

This work is licensed under the Creative Commons Attribution International License (CC BY 4.0).

http://creativecommons.org/licenses/by/4.0/

\section{(c) (i) Open Access}

\section{ABSTRACT}

Humans depend on the coordinated activity of their lower extremities for mobility, an essential feature of Homo sapiens. In addition, they use vision to use this mobility to successfully navigate through their environment. During development, mobility appears to mature first, and then it is coordinated with navigation. Thus, the two, mobility and navigation are likely inter-dependent in function. Recent studies have indicated that compromising the integrity of the knee, a central element of the lower extremity motion segment, can lead to molecular alterations in both the cornea including the central cornea where light passes, as well as the interior of the eye (the vitreous humor). Not all insults to the knee lead to reproducible alterations in the eye, indicating some specificity in the response. In addition, it was recently reported that alterations to the cells in the vitreous humor occur following dietary induction of obesity in a rat model. As humans with obesity, as well as arthritis of the knee are at risk for ocular involvement and exhibit altered gait characteristics, the clinical and preclinical data raise the possibility of a "knee-eye-brain axis" to control or regulate mobility and navigation. Better delineation of such an axis could have implications for variations in control during maturation, and well as during aging when vision and mobility can be compromised, with increased risk for serious falls and failure to successfully navigate the environment.

\section{INTRODUCTION-MOBILITY}

As mammals evolved on Earth, to function on land required the maturation of effective mobility in 
the $1 \mathrm{~g}$ environment of Earth. This required formation of sophisticated appendages such as legs and arms that could articulate. As humans appear to have evolved from animals that moved primarily with four limbs on the ground, the development of the ability to function upright required evolution of a more sophisticated system, with the arms (fingers, hands, and elbows) now taking on different roles from walking/running, and the lower extremities evolving to support the trunk/spine and head effectively. The drive to walk/be mobile in an upright configuration must serve a survival advantage (i.e. seeing and responding to predators or threats more effectively over the savanna grasses, ability to detect prey that would otherwise be hidden). Thus, survival required effective mobility, which in the case of humans meant development of a strong and coordinated lower limb system (i.e. motion segments consisting of feet, ankles, knees, hips, pelvis, and associated muscles, tendons, bones, and ligaments, etc). All of the tissues, except for adult articular cartilage of articulating joints are innervated, and the driver of all of the motions is the muscles controlled by neural input to generate forces. Not only is coordination required at the motion segment level, but as bipedal animal, coordination between the lower limbs are required for effective mobility.

Within an articulating joint of a motion segment, such as the knee, these joints are proposed to function as an organ system [1-3]. That is, to understand how the joint is working, and the interdependence of the parts, requires one to look at the knee for instance as an integrated unit. Thus, following an injury to one component requires adaptation of the other parts, which if unsuccessful, leads to loss of function, pain and disability [2]. Furthermore, disease such as osteoarthritis, long portrayed as a cartilage disease, is really a disease of this organ system [3].

As mentioned above, both lower limbs of a human must work in a coordinated manner to maintain optimal functioning. Thus, there must be mechanisms controlling interaction between the two lower limbs and coordination of movement. This coordination is likely accomplished at the level of the dorsal root ganglion of the spine, likely with additional neural input from the brain. Such cross talk between lower limbs has been shown in preclinical models, and concluded from some human conditions. Thus, in rabbits it has been reported that injury to ligaments of the knee of one leg leads to alterations in the contralateral knee [4]. Similarly in rabbits, injection of the quadriceps muscle complex of one leg with Botulium toxin leads to atrophy of the muscle of the injected leg with subsequent development of fibrosis and accumulation of fat in the muscle [5]. Interestingly, the corresponding muscle of the contralateral leg undergoes fibrosis, but without atrophy and fat accumulation $[5,6]$. Thus, in this model some of the changes in the injected muscle are also evident in the opposite leg, but not all. This result indicates that the underlying mechanisms for the contralateral effect are mediated via signals that can recapitulate some but not all of the features of the injected tissue. Finally, in a mouse model, it was shown that a stab injury to the Achilles tendon of one leg led to accelerated ossification of the tissue, and this was also evident in the contralateral Achilles tendon [7]. While not as defined in humans, it is noteworthy that inflammatory arthritis (rheumatoid arthritis, RA) is usually a bilateral disease. It may start in one knee, ankle, hand, but then often involves the contralateral limb [8]. One mechanism by which this could happen is via the contralateral effect, with possibly neural signals leaving the initially affected inflamed joint, with information traveling to the DRG, and then down to the contralateral joint $[8,9]$. Once in the contralateral joint, release of neurotransmitters such as Substance P could induce acute neuro-inflammation which then becomes chronic. Substance P can also influence gene expression in endogenous cells of the joint (i.e. fibroblasts) [10], as well cells in ligaments [11], in the synovium [12] and elsewhere, to initiate the response pattern leading to chronic inflammation and other features of RA.

\section{MOBILITY + NAVIGATION}

From the above discussion, it is clear that the physical act of mobility and its control is complex. Another level of complexity is the functional aspect of using the mobility to traverse the environment in a safe manner, avoiding obstacles and predators, as well as providing directionality to the mobility. In addition, one needs to maintain an upright positioning and maintain head movement control, as well as control energy expenditures. Thus, this requires integration between the physical act of mobility, which involves 
neural control at multiple levels, and a navigation system. Therefore, humans primarily depend on their eyes and sight for navigation, and likely other cues from hearing.

Studies assessing gait of individuals with sight and then blindfolded have indicated that humans use sight for maintenance of posture $[13,14]$ and aspects of mobility $[15,16]$. Interestingly, such experiments with obese individuals can lead to more pronounced deviations after being blindfolded [14]. Additionally, with aging, vision integrity can start to fail in many individuals, with development of overt diseases of the ocular system (i.e. macular degeneration; cataracts), resulting in increased risks for falls and failure to navigate the environment effectively.

Navigation can also be compromised by diseases affecting brain areas that impact motor control and neural control of movement. Thus, conditions such as multiple sclerosis [17], Parkinson Disease [18], and even cognitive disorders such as Alzheimer's disease [19] can affect both navigation and mobility. Thus, diseases and alterations to the integrity of the eye can compromise navigation.

Therefore, navigation of the environment via mobility is likely controlled at the level of the motion segment, the ocular system, and centres/areas in the brain. Breakdown of the integrity of the system can occur at the level of elements of the motion segment (i.e. development of diseases such as osteoarthritis, osteoporosis, sarcopenia), the ocular system, or elements of the brain that exert neural control of both navigation and the motion segment. Work by Salo and Tatton [20] have shown that in some strains of mice, age-related loss of innervation of a joint such as a knee, can increase risk for osteoarthritis, with loss of knee integrity. Whether such loss of innervation would potentially abrogate links to the eye is not known. Injury to brain centres via a stroke can also lead to loss of mobility in a specific motion segment, and blindness can obviously drastically compromise the ability to navigate the environment. Thus, control of both navigation and mobility is a complex integrated "axis" consisting of "mobility-vision-brain" elements.

\section{JOINT INJURY CAN AFFECT THE CORNEA OF THE EYE}

From the above discussion, it is clear that motion and navigation are interrelated and interdependent functionally. The question then arises "are the eye, the navigator, and the knee, a central element for motion, interrelated biologically"? If so, then what mechanisms might be involved and responsible for control?

Studies performed nearly a decade ago indicated that injury to the knee of a rabbit, namely transection of the anterior cruciate ligament (ACL), led to gene expression alterations in the rabbit cornea [21]. The alterations in the cornea differed between the central part of the cornea through which light passes, and the periphery of the cornea which serves more of a supportive role. Both parts are collagen-based connective tissues, but the collagen in the central part is different from that of the periphery [21, 22]. Interestingly, the molecular changes detected in the cornea were evident by 3 weeks post-knee injury, and persisted past 6 weeks post-injury [21]. If the animals were injected locally in the knee, or systemically via a subcutaneous injection with a glucocorticoid (GC), the alterations to homeostasis in the cornea were abolished if the GC was injected at 3 weeks post-injury, but not if the injection was delayed to 6 weeks post-injury [21].

As the ACL is an intra-articular ligament, we next wanted to determine whether injury to an extra-articular ligament of the knee would also lead to alterations in the cornea of the eye. For this study the well-established medial collateral ligament (MCL) model was chosen. Following bilateral MCL transection, consistent molecular changes were again detected in the peripheral and central portions of the cornea [22]. Interestingly, the changes induced by the MCL transection were somewhat different from those detected after the ACL transection, potentially indicating that the location of the injury can influence the actual changes to the corneal cells.

As the cornea functions to both contain the interior of the globe of the eye and serve as a barrier, as well as permit light to pass into the eye, it must be lubricated by fluid from the lacrimal glands [23]. The function of the lacrimal glands is influenced by sex hormones in females, and post-menopausal females 
often suffer from dry eye syndrome which can affect the cornea [24-26]. Thus, injury to the knee could be affecting the cornea directly, or indirectly via alterations to the lacrimal glands. To address this issue, female rabbits were subjected to ovariohysterectomy (OHX) +/- bilateral MCL injury, and then the cornea were assessed. The results indicated that the molecular changes to the cornea after OHX or MCL injury were nearly identical [27], but still different from the changes observed after ACL injury. Therefore, following injury to the extra-articular MCL, the corneal changes may be due in part, to impact on the lacrimal glands rather than a direct influence on cells of the cornea.

From these studies, a knee injury can impact the surface structures of the eye, but to date it is not known whether the changes detected alter the cornea so as to influence the navigator function of the eye. The changes could influence the fidelity of light passing into the eye, but that remains to be confirmed.

How such a knee injury could impact the cornea also remains unknown. It could be due to release of soluble mediators that leave the site of injury and travel via the systemic circulation to impact the cornea directly, or indirectly via the lacrimal glands. Alternatively, it could be due to an impact on nerves in the knee to send information via neural circuitry to directly to the eye or via the lacrimal glands [28]. As the impact of the intra-articular injury to the ACL was different from that of the extra-articular injury to the MCL, it could be due to either mechanism depending on where the knee injury occurred.

\section{LOSS OF KNEE INTEGRITY CAN IMPACT THE INTERIOR OF THE EYE}

To address the issue of whether loss of knee integrity can influence homeostasis of the interior of the eye, additional sets of studies were performed (Hart et al., in preparation). The first set was focused on inducing unilateral (right knee) inflammatory arthritis in the female rabbit knee using an antigen-induced arthritis protocol. In this model, rabbits are systemically immunized with a protein antigen (ovalbumin) and then challenged via intra-articular injection with soluble antigen. At 7, 14 and 28 days post challenge, the knees were assessed for inflammation, and the vitreous humour (VH) and vitreous humor cells (VHC) were removed aseptically. The VHC were analyzed for mRNA expression levels for a variety of inflammatory mediators and growth factors. This compartment was chosen as the interior of the eye is an immune privileged site [29-32], and alterations to this site could lead to subsequent alterations at the level of the retina. For these studies, the left and right eyes were kept separate in an attempt to ascertain whether the right eye may be affected sooner than the left one. The results did not detect an eye preference for initiation of the VH cell changes (data not shown), and thus, it was concluded that unilateral knee injury led to bilateral changes in the $\mathrm{VH}$ cells.

Potentially relevant to the above discussion, in humans, development of juvenile inflammatory arthritis (JIA) can lead to increased risk for uveitis, an inflammatory condition of the anterior part of the eye [33], as well as other alterations to ocular structures [34]. If left untreated, it can result in blindness. Of those with JIA, the patients at highest risk for eye involvement are those with knee arthritis, and do not have a high number of other joints involved. There are also age-dependent sex differences in the incidence of uveitis in JIA $[35,36]$. While this connection between the knee and the eye is possibly consistent with a "knee-eye axis" related to mobility and navigation, literature regarding kinematic or gait abnormalities in JIA is somewhat limited [37-39]. Also, based on the methods used, it is not possible to distinguish variations in gait due to the arthritis itself, or whether those with JIA + uveitis exhibit a unique pattern of disturbance. Furthermore, while a subset of JIA patients are at increased risk for eye involvement, many of the same subset of JIA patients do not exhibitovert eye involvement. Thus, either there is something unique about the actual arthritis in the affected subset, some additional genetic predisposition is required to manifest the eye involvement, or it is a result of some specific environmental interaction which manifests as uveitis in this subset. As JIA is a chronic disease, but the uveitis is may be episodic, no direct link between the knee and eye, as might be expected from a "knee-eye axis", can be concluded at the present time from such patients. However, it is also possible that changes to vision in such patients leads to compromise of the navigation function without the appearance of overt disease. 


\section{POTENTIAL ALTERATIONS TO VISION WITH OBESITY}

Individuals with obesity are reported to be at risk for development of some ocular diseases such as macular degeneration $[40,41]$ and others $[42,43]$. However, it is not clear whether this risk is related to a direct effect of the fat, the dysregulated inflammatory state, or a secondary consequence of obesity related to T2D development, for example. As the eye is an immune privileged site [29-32], disruption of homeostasis via a dysregulated inflammatory system could contribute to loss of such privilege and allow for inappropriate inflammatory or immune activities within the eye (e.g. via the vitreous humor), as well as at the level of the cornea. Recently, we have reported that following induction of diet-induced obesity, the gene expression patterns are altered in cells of the vitreous humor $(\mathrm{VH})$, and that via assessment of $\mathrm{VH}$ proteins via the Luminex platform protein array methodology, the protein homeostasis of this fluid is disrupted with the elevated presence of inflammatory mediators [44]. While we have not yet correlated these ocular changes with overt disease processes in the preclinical model, disruption of homeostasis in this compartment could lead to compromise in the navigator status of the eye. In addition, it is not yet possible to directly attribute the changes in the $\mathrm{VH}$ of the DIO rats to the systemic inflammatory alterations detected. Such changes could also be an indirect effect of the joint damage occurring after induction of DIO [45]. Since the current rat studies used only the 12 week time point post-DIO to assess joint damage and the $\mathrm{VH}$ alterations, it is not yet known whether the joint damage preceded the alterations in the eye, they developed in parallel, or the eye alterations preceded the joint damage. Resolution of this issue is the subject of current research.

\section{SUMMARY}

This issue of eye involvement in obesity is likely very clinically relevant as humans use their lower extremities for mobility, with the knee a central component of the motion segments, but use their eyes to navigate through their environment. As such, mobility is critical, but navigation even more so. However, it is not yet known whether injury to the ankle or the hip also leads to changes in the eye, or whether there may be a specific "knee-eye" axis. This is an area of active research, as is determining whether this potential axis is unidirectional (knee to eye) or bidirectional (also eye to knee). Thus, mobility may be intrinsically integrated with navigation, but details regarding the integration of such integration are still lacking and in need of further research. While learning to walk, considerable effort is spent learning to coordinate mobility and navigation, usually, with mobility occurring first, and then successful navigation occurring secondarily. Watching a young human learn to walk, and then to navigate indicates the amount of effort that is expended to master both aspects of function. Whether the integration occurs optimally in all people is also likely an open question. Many individuals, both children and adults have been labeled as "clumsy", but whether the basis for such a label resides in specific vision defects related to navigation, to a less than optimal integration of mobility and navigation circuits, or some other explanation remains to be defined.

However, skeletally mature adult humans define where they are in space while they are mobile without actually overtly thinking about it, but may require more active thinking with aging as the systems involved decline in integrity. This latter aspect of mobility likely involves brain elements associated with control of eye movement [46], memory, or other specific areas of the brain such as the hypothalamus [47]. Relevant to this discussion, Berenbaum and Meng [48] proposed a "brain-joint axis" in osteoarthritis, and it involved the suprachiasmatic nucleus of the hypothalamus that is responsible for circadian clock activity. Interestingly, as discussed above, assessment of both normal body weight individuals and those with obesity blindfolded have reported that such interruption of the "knee-eye" axis leads to abnormal gait patterns, with the alterations more pronounced in those with obesity. Therefore, potential compromise of ocular integrity via chronic inflammatory changes such as those with obesity or after a knee injury may have more impact on the fidelity of mobility in both new and established patterns of movement, even in the context of walking in a familiar environment with some brain elements intact. This may reflect the central nature of a "knee-eye-brain/neural axis" that needs to be intact to optimize both mobility and navigation. As all knee joint tissues (and other joints of the body) except for articular cartilage is inner- 
vated, and muscles are dependent on neuro-activation to initiate force generation, the integrity of such an axis of control/regulation may be necessary for optimal mobility and navigation, and obesity and/or lower extremity joint injury, and their disrupted inflammatory/neural state can be considered a threat to such integrity at several points (i.e. muscles, joint integrity, loss of visual integrity). Thus, this area, as well as those related to "motion segment-eye interdependence relationships" is in need of further investigation, and preclinical models such as the rat and rabbit models offer the ability to study such regulatory circuits in detail.

\section{ACKNOWLEDGEMENTS}

The author thanks the many colleagues, trainees, and laboratory staff over the past several years for conversations on this topic, as well as those who have contributed studies that have helped shape the ideas presented in this article.

\section{REFERENCES}

1. Lo, I.K., et al. (2003) Structure and Function of Diarthrodial Joints. In: McGinty, J.B., Ed., Operative Arthroscopy, 3rd Edition, Lippincott, Williams and Wilkins, Philadelphia, 41-126.

2. Frank, C.B., et al. (2004) New Perspectives on Bioengineering of Joint Tissues: Joint Adaptation Creates a Moving Target for Engineering Replacement Tissues. Annals of Biomedical Engineering, 32, 458-465. https://doi.org/10.1023/B:ABME.0000017548.85451.b7

3. Loeser, R.F., Goldring, S.R., Scanzello, C.R. and Goldring, M.B. (2012) Osteoarthritis: A Disease of the Joint as an Organ. Arthritis Rheum, 64, 1697-1707. https://doi.org/10.1002/art.34453

4. Frank, C.B., Loitz, B., Bray, R., Chimich, D., King, G. and Shrive, N. (1994) Abnormality of the Contralateral Ligament after Injuries of the Medial Collateral Ligament. An Experimental Study in Rabbits. The Journal of Bone and Joint Surgery-American Volume, 76, 403-412. https://doi.org/10.2106/00004623-199403000-00011

5. Leumann, A., Longino, D., Fortuna, R., Leonard, T., Vaz, M.A., Hart, D.A. and Herzog, W. (2012) Altered Cell Metabolism in Tissues of the Knee Joint in a Rabbit Model of Botulinum Toxin A-Induced Quadriceps Muscle Weakness. Scandinavian Journal of Medicine \& Science in Sports, 22, 776-782. https://doi.org/10.1111/j.1600-0838.2011.01309.x

6. Fortuna, R., et al. (2015) A Clinically Relevant BTX-A Injection Protocol Leads to Persistent Weakness, Contractile Material Loss, and an Altered mRNA Expression Phenotype in Rabbit Quadriceps Muscles. Journal of Biomechanics, 48, 1700-1706. https://doi.org/10.1016/j.jbiomech.2015.05.018

7. O`Brien, E.J., Shrive, N.G., Rosvold, J.M., Thornton, G.M., Frank, C.B. and Hart, D.A. (2013) Tendon Mineralization is Accelerated Bilaterally and Creep of Contralateral Tendons Is Increased after Unilateral Needle Injury of Murine Achilles Tendons. Journal of Orthopaedic Research, 31, 1520-1528.

https://doi.org/10.1002/jor.22404

8. Levine, J.D., et al. (1987) Contribution of the Nervous System to the Pathophysiology of Rheumatoid Arthritis and Other Polyarthritides. Rheumatic Disease Clinics of North America, 13, 369-383.

9. Decaris, E., Guingamp, C., Chat, M., et al. (1999) Evidence for Neurogenic Transmission Inducing Degenerative Cartilage Damage Distant from Local Inflammation. Arthritis \& Rheumatology, 42, 1951-1560. https://doi.org/10.1002/1529-0131(199909)42:9<1951::AID-ANR22>3.0.CO;2-D

10. Murphy, P.G. and Hart, D.A. (1993) Plasminogen Activators and Plasminogen Activator Inhibitors in Connectives Tissues and Connective Tissue Cells: Influence of the Neuropeptide Substance P on Expression. Biochimica Biophysica Acta, 1182, 205-214. https://doi.org/10.1016/0925-4439(93)90142-N

11. Salo, P., et al. (2007) Neuropeptides Regulate Expression of Matrix Molecule, Growth Factor and Inflammatory 
Mediator mRNA in Explants of Normal and Healing Medial Collateral Ligament. Regulatory Peptides, 142, 1-6. https://doi.org/10.1016/j.regpep.2007.01.001

12. Keeble, J.E. and Brain, S.D. (2004) A Role for Substance P in Arthritis? Neuroscience Letters, 361, 176-179. https://doi.org/10.1016/j.neulet.2003.12.020

13. Hue, O., et al. (2007) Body Weight Is a Strong Predictor of Postural Stability. Gait Posture, 26, 32-38. https://doi.org/10.1016/j.gaitpost.2006.07.005

14. D'Hondt, E., et al. (2011) The Role of Vision in Obese and Normal-Weight Children's Gait Control. Gait Posture, 33, 179-184. https://doi.org/10.1016/j.gaitpost.2010.10.090

15. Hallemans, A., et al. (2009) Visual Deprivation Leads to Gait Adaptations That Are Age- and Context-Specific: II. Kinematic Parameters. Gait Posture, 30, 307-311. https://doi.org/10.1016/j.gaitpost.2009.05.017

16. Thompson, J.D. and Franz, J.R. (2017) Do Kinematic Metrics of Walking Balance Adapt to Perturbed Optical Flow? Human Movement Science, 54, 34-40. https://doi.org/10.1016/j.humov.2017.03.004

17. Comber, L., et al. (2017) Gait Deficits in People with Multiple Sclerosis: A Systematic Review and Meta-Analysis. Gait Posture, 51, 25-35. https://doi.org/10.1016/j.gaitpost.2016.09.026

18. Amano, S., et al. (2013) Ambulation and Parkinson Disease. Physical Medicine and Rehabilitation Clinics of North America, 24, 371-392. https://doi.org/10.1016/j.pmr.2012.11.003

19. Allali, G., et al. (2016) Gait Phenotype from Mild Cognitive Impairment to Moderate Dementia: Results from the GOOD Initiative. European Journal of Neurology, 23, 527-541. https://doi.org/10.1111/ene.12882

20. Salo, P.T. and Tatton, W.G. (1993) Age-Related Loss of Knee Joint Afferents in Mice. Journal of Neuroscience Research, 35, 664-677. https://doi.org/10.1002/jnr.490350609

21. Kydd, A.S., et al. (2007) Impact of Age, Systemic Glucocorticoids, and Progressive Knee Arthritis on Specific mRNA Levels in Different Areas of the Rabbit Cornea. Cornea, 26, 352-361. https://doi.org/10.1097/ICO.0b013e318033a534

22. Achari, Y., et al. (2008) Influence of Timing (Pre-Puberty or Skeletal Maturity) of Ovariohysterectomy on mRNA Levels in Corneal Tissues of Female Rabbits. Molecular Vision, 14, 443-455.

23. Tiffany, J.M. (2008) The Normal Tear Film. Developments in Ophthalmology, 41, 1-20. https://doi.org/10.1159/000131066

24. Stern, M.E., et al. (1998) The Pathology of Dry Eye: The Interaction Between the Ocular Surface and Lacrimal Glands. Cornea, 17, 584-589. https://doi.org/10.1097/00003226-199811000-00002

25. Versura, P., et al. (2015) Sex-Steroid Imbalance in Females and Dry-Eye. Current Eye Research, 40, 162-175. https://doi.org/10.3109/02713683.2014.966847

26. Sriprasert, I., et al. (2016) Dry Eye in Postmenopausal Women: A Hormonal Disorder. Menopause, 23, 343-351. https://doi.org/10.1097/GME.0000000000000530

27. Achari, Y., et al. (2010) Influence of Bilateral Medial Collateral Ligament Injury on mRNA Expression in Distal Corneal Tissues of Control and Ovariohysterectomized Rabbits. Cornea, 29, 418-431. https://doi.org/10.1097/ICO.0b013e3181bd45ec

28. Dartt, D.A. (2009) Neural Regulation of Lacrimal Gland Secretory Processes: Relevance in Dry Eye Diseases. Progress in Retinal and Eye Research, 28, 155-177. https://doi.org/10.1016/j.preteyeres.2009.04.003

29. Niederkorn, J.Y. (2002) Immune Privilege in the Anterior Chamber of the Eye. Critical Reviews in Immunology, 22, 13-46. https://doi.org/10.1615/CritRevImmunol.v22.i1.20

30. Niederkorn, J.Y. (2007) The Induction of Anterior Chamber-Associated Immune Deviation. Chemical Immunology and Allergy, 92, 27-35. https://doi.org/10.1159/000099251 
31. Streilein, J.W. and Stein-Streilein, J. (2000) Does Innate Immune Privilege Exist. Journal of Leukocyte Biology, 67, 479-487. https://doi.org/10.1002/jlb.67.4.479

32. Stein-Streilein, J. and Streilein, J.W. (2002) Anterior Chamber Associated Immune Deviation (ACAID): Regulation, Biological Relevance, and Implications for Therapy. International Reviews of Immunology, 21, 123-152. https://doi.org/10.1080/08830180212066

33. Clarke, S.L., et al. (2016) Juvenile Idiopathic Arthritis-Associated Uveitis. Pediatric Rheumatology Online Journal, 14, 27. https://doi.org/10.1186/s12969-016-0088-2

34. Paroli, M.P., et al. (2013) Severe Macular Edema in Patients with Juvenile Idiopathic Arthritis-Related Uveitis. Case Reports in Ophthalmological Medicine, 2013, Article ID: 803989. https://doi.org/10.1155/2013/803989

35. Saurenmann, R.K., et al. (2010) Risk Factors for Development of Uveitis Differ between Girls and Boys with Juvenile Idiopathic Arthritis. Arthritis \& Rheumatology, 62, 1824-1828. https://doi.org/10.1002/art.27416

36. Qian, Y. and Acharya, N.R. (2010) Juvenile Idiopathic Arthritis-Associated Uveitis. Current Opinion in Ophthalmology, 21, 468-472. https://doi.org/10.1097/ICU.0b013e32833eab83

37. Choban, S. and Killian, J.T. (1990) Evaluation of Acute Gait Abnormalities in Preschool Children. Journal of Pediatric Orthopaedics, 10, 74-78. https://doi.org/10.1097/01241398-199001000-00014

38. Fairburn, P.S., et al. (2002) The Use of Multidisciplinary Assessment and Scientific Measurement in Advanced Juvenile Idiopathic Arthritis Can Categorize Gait Deviations to Guide Treatment. Archives of Disease in Childhood, 87, 160-165. https://doi.org/10.1136/adc.87.2.160

39. Hartmann, M., et al. (2010) Effects of Juvenile Idiopathic Arthritis on Kinematics and Kinetics of the Lower Extremities Call for Consequences in Physical Activities Recommendations. International Journal of Pediatrics, 2010, Article ID: 835984. https://doi.org/10.1155/2010/835984

40. Cheung, L.K. and Eaton, A. (2013) Age-Related Macular Degeneration. Pharmacotherapy, 33, 838-855. https://doi.org/10.1002/phar.1264

41. Zhang, Q.Y., et al. (2016) Overweight, Obesity, and Risk of Age-Related Macular Degeneration. Investigative Ophthalmology \& Visual Science, 57, 1276-1283. https://doi.org/10.1167/iovs.15-18637

42. Merlotti, C., et al. (2017) Bariatric Surgery and Diabetic Retinopathy: A Systemic Review and Meta-Analysis of Controlled Clinical Studies. Obesity Reviews, 18, 309-316. https://doi.org/10.1111/obr.12490

43. Tham, Y.C. and Cheng, C.Y. (2017) Associations between Chronic Systemic Diseases and Primary Open angle Glaucoma: An Epidemiological Perspective. Clinical \& Experimental Ophthalmology, 45, 24-32. https://doi.org/10.1111/ceo.12763

44. Collins, K.H., et al. (2017) Diet-Induced Obesity Leads to Pro-Inflammatory Alterations to the Vitreous Humour of the Eye in a Rat Model. Inflammation Research, 67, 139-146. https://doi.org/10.1007/s00011-017-1102-y

45. Collins, K.H., et al. (2018) High Fat High-Sucrose Diet-Induced Obesity Results in Joint-Specific Development of Osteoarthritis-Like Degeneration in a Rat Model. Bone \& Joint Research.

46. Baird-Gunning, J.J.D. and Lueck, C.J. (2018) Central Control of Eye Movements. Current Opinion in Neurology, 31, 90-95.

47. Trachtman, J.N. (2010) Vision and the Hypothalamus. Optometry, 81, 100-115. https://doi.org/10.1016/j.optm.2009.07.016

48. Berenbaum, F. and Meng, O.-J. (2016) The Brain-Joint Axis in Osteoarthritis: Nerves, Circadian Clocks and Beyond. Nature Reviews Rheumatology, 12, 508-516. https://doi.org/10.1038/nrrheum.2016.93 